\title{
APORTES DE LA PSICOLOGÍA JURÍDICA A LOS PROCESOS DE ACOMPAÑAMIENTO PSICOSOCIAL A LAS VICTIMAS DE LA VIOLENCIA EN EL DEPARTAMENTO DEL MAGDALENA
}

\author{
Cindy Tobías Loaiza, Cindy Muñoz Velázquez y Madeleide Ricaurte Fuentes*
}

Fecha de recibido: 31 de octubre de 2011

Fecha de aprobación: 7 de diciembre de 2011

Artículo resultado de Investigación

\begin{abstract}
Resumen
Las víctimas de la violencia reciben apoyo por parte del Estado para obtener acompañamiento psicosocial y reparar el daño sufrido. A pesar de los innegables avances normativos materializados en el marco de la ley de víctimas, estas corren el riesgo de ser revictimizadas. El presente informe de investigación recurre a metodologías cualitativas y a la psicología jurídica para determinar las mejores prácticas en la orientación de los procesos de acompañamiento psicosocial a víctimas de la violencia. La población objeto de estudio se encuentra en el Departamento del Magdalena.
\end{abstract}

\section{Palabras clave}

Psicología jurídica, víctimas de la violencia, acompañamiento psicosocial, empoderamiento.

\section{LEGAL PSYCHOLOGY'S CONTRIBUTIONS TO THE PROCESSES OF PSYCHOSOCIAL SUPPORT FOR VICTIMS OF VIOLENCE IN THE DEPARTMENT OF MAGDALENA}

\begin{abstract}
The victims of the violence receive support by part of the State to obtain accompaniment psicosocial and repair the damage. In spite of the innegables advance normative materialized in the frame of the law of victims, these run the risk to be revictimizadas. In spite of the undeniable advances normatives materialized in the framework of the law of victims, these have the risk to be revictimized. The present report of investigation draws on qualitative methodologies and legal psychology to identify best practices in guiding the processes of psychosocial support to victims of violence. The target population of study is in the Department of Magdalena.
\end{abstract}

\section{Keywords}

Legal psychology, victims of violence, psychosocial support, empowerment.

* Estudiantes del pregrado en Psicología de la Universidad del Magdalena - Santa Marta Magdalena. (con culminación de materias) Semilleros de investigación en calidad de auxiliares de investigación en temas de psicología jurídica y empoderamiento de victimas, bajo la dirección y asesoría del docente Investigador Edimer Leonardo Latorre Iglesias, C. Ph.D, quien es director del grupo de investigación Comunicación y Sociedad de la Universidad Sergio Arboleda. Correos electrónicos: cipatolo_04@ hotmail.com ; cindymimuve_14@hotmail.com ; maderifu9@hotmail.com ; edimerlatorre@hotmail.com 


\title{
CONTRIBUIÇÕES DA PSICOLOGIA LEGAL PARA OS PROCESSOS DE APOIO PSICOSSOCIAL ÀS VÍTIMAS DE VIOLÊNCIA NO DEPARTAMENTO DE MADALENA
}

\section{Resumo}

\begin{abstract}
As vítimas da violência recebem apoio por parte do Estado para obter acompañamiento psicosocial e consertar o dano sofrido. Apesar dos inegáveis avanços incorporados no quadro regulamentar da lei de vítimas, eles correm o risco de ser re-vitimadas. Este relatório de investigação baseia-se em metodologias qualitativas e à psicologia legal para identificar as melhores práticas na orientação os processos de apoio psicossocial às vítimas de violência. A população em estudo é do Departamento de Magdalena.
\end{abstract}

\section{Palavras-chave}

Psicologia juridica, vítimas de violência, apoio psicossocial, empowerment.

\section{INTRODUCCIÓN}

Los eventos legislativos conllevan la interpretación de circunstancias particulares que deben ser solucionadas desde el campo normativo, bajo la estrictez de la legalidad y la legitimidad, interesada por igual en la solución de la problemática que acompaña a la victima por su esencialidad, originando procesos de diversa índole y dimensión que deben ser abordados científicamente.

Dentro de esos procesos encontramos el acompañamiento psicosocial a las víctimas de la violencia en Colombia, estructurados en torno al resarcimiento integral y holístico de lo padecido por las víctimas.

La victimología, como una de las especialidades de la psicología jurídica, se orienta al estudio y gestión de la prevención y del tratamiento de los procesos que conllevan el restablecimiento de las condiciones de quienes han sido sujetos pasivos de circunstancias que les cambian las expectativas de vida. Permite la intervención en estados de crisis, su asesoría, su protección, su rehabilitación e incorporación social.

Esto relieva la importancia en la utilización de metodologías cualitativas como herramienta en la investigación, para determinar las realidades en las cuales se suscita el fenómeno y respeta la contextualidad a la cual se circunscribe, para luego, con un abordaje sinérgico desde la Psicología Jurídica, exponer la conducta y el comportamiento humano, respecto de la dinámica jurídica, que debe estar orientada al mejoramiento psicosocial de los afectados.

Este proyecto es un informe de avance preliminar, que condensa la aproximación teórica y metodológica realizada, en espera de corroborar empíricamente los presupuestos teóricos que guían el presente estudio.

\section{DEFINICIÓN DEL PROBLEMA Y PRECISIÓN EN CUANTO AL MÉTODO}

Para el abordaje del problema es pertinente el planteamiento de Elizabeth Lira Kornfeld (2000, p. 187) frente a lo traumático que evidencia la experiencia de ser víctima:

Lo traumático esta dado por dos elementos simultáneos: el impacto sorpresivo e inesperado de amenazas vitales múltiples las que, al mismo tiempo que aparecen como previsibles, son difíciles de discriminar, evitar o enfrentar. El segundo elemento surge de la desorganización experimentada por los sujetos concretos, las familias, los grupos sociales, los partidos políticos, etc. Esta desorganización conduce a respuestas inicialmente caóticas o inefectivas, que 
incluso aumentan el carácter traumático de la experiencia. La descripción de la realidad social desde la óptica de lo traumático implica que con cierta regularidad los hechos sobrepasan la capacidad y los recursos de los grupos sociales, o de las personas involucradas, los que, incapaces de vivir en forma permanente en situaciones insostenibles, realizan ajustes destructivos o autodestructivos, que se hacen crónicos y que conducen más bien al deterioro psicológico y social.

Deriva en tema de gran importancia, la gestión legal para restablecer las condiciones sociales producto de la violencia. En Colombia se intenta con la ley de Victimas y su estructura normativa pretende la protección a las víctimas, existiendo un alto riesgo en el tratamiento estandarizado y masificado, impidiendo con ello el estudio personalizado de las condiciones individuales $e$ irrepetibles que deben orientar la acción del Estado a desarrollar centros de atención según las condiciones de las mismas, en especial a sus características particulares, que permitan la reorganización interna del sujeto y un empoderamiento muy particular para que este asuma su proyecto vital y como lo plantea Martin-Baro (2000, p. 78):

Pero al hablar de trauma psicosocial se quieren subrayar también otros dos aspectos, que con frecuencia tienden a olvidarse: (a) que la herida que afecta a las personas ha sido producida socialmente, es decir, que sus raíces no se encuentran en el individuo, sino en su sociedad, y (b) que su misma naturaleza se alimenta y mantiene en la relación entre el individuo y la sociedad, a través de diversas mediaciones institucionales, grupales $e$ incluso individuales. Lo cual tiene obvias e importantes consecuencias a la hora de determinar que debe hacerse para superar esos traumas.

El marco normativo en el cual se sostiene la Ley de Victimas, es el de la justicia transicional, que propende por la trascendencia del conflicto, haciendo hincapié en la reparación económica, en la restitución de las tierras y en el acompañamiento psicológico a la víctima.

De ahí la necesidad de encontrar formas de empoderar a las víctimas para que estas asuman su dolor y sean capaces de reconstruirse desde adentro, pero también de reconstruir psicosocialmente su fragmentado entorno, tal y como lo precisa Gutiérrez (2011, p. 5):

Así pues, toda justicia debe partir de la víctima, de sus necesidades y deseos, garantizando que las decisiones que las personas que han sido puesta en situación de víctimas sean respetadas. La justicia debe buscar que las personas que alguna vez fueron victimizadas (victimas de algún tipo de delito o agresión) y revictimizadas (mala atención por parte de quienes administran justicia, etiquetamiento social, etc) sientan que son realmente las protagonistas, que la agresión que padecieron no impedirá que puedan seguir actuando con el control de sus vidas, que puedan salir a la calle con la frente en alto, seguras, confiadas, con la sensación de que pudieron hacer algo por ellas mismas con la protección del Estado $y$ de su comunidad, que encontraron redes sociales de apoyo, que no fueron invisibles, que su voz fue escuchada y su dolor reparado.

De este conjunto de ideas surge la pregunta problema que orientara el proceso de investigación: ¿Cuál es el aporte de la psicología jurídica a los procesos de acompañamiento psicosocial a las víctimas de la violencia en el Departamento del Magdalena?

Siendo el objetivo general: Determinar los principales aportes de la psicología jurídica a los procesos de acompañamiento psicosocial de las víctimas de la violencia en el Departamento del Magdalena. De este gran objetivo general desprendemos los siguientes objetivos particulares: 
- Determinar los procesos de acompañamiento psico-jurídico a las víctimas en el Departamento del Magdalena.

- Realizar una clasificación de las victimas en el Departamento del Magdalena.

- Escoger una muestra representativa de victimas en el Departamento del Magdalena.

- Evaluar los alcances actuales de la atención a las víctimas en el Departamento del Magdalena.

- Precisar el aporte teórico-práctico de la psicología jurídica a los procesos acompañamiento psicosocial de las víctimas de la violencia en el Departamento del Magdalena.

La investigación se orienta bajo la egida del paradigma cualitativo, descrita desde la óptica de Bonilla y Rodríguez (2005, p. 119):

(...) hacer una aproximación global de las situaciones sociales para explorarlas, describirlas y comprenderlas de manera inductiva. Es decir, a partir de los conocimientos que tienen las diferentes personas involucradas en ellas y no deductivamente, con base en hipótesis formuladas por el investigador externo. Esto supone que los individuos interactúan con los otros miembros de su contexto social compartiendo el significado y el conocimiento que tienen de sí mismo y de su realidad.

Se pretende a través de la construcción de grupos focales y de entrevistas en profundidad con las victimas que conforman las muestras representativas, en los lugares donde se dieron grandes hechos de violencia, estudiar los temas pertinentes partiendo de los diferentes contextos sociales, los diversos procesos de acompañamiento psicosocial de los sujetos y las comunidades, como también los diversos recursos interiores y exteriores.
Se escoge la herramienta de grupos focales dado que estos, según Janice Morse (2003, p. 263) proporcionan:

(...) luces en cuanto las creencias y actitudes que subyacen al comportamiento. Los datos relacionados con las percepciones $y$ opiniones se enriquecen por medio de la interacción del grupo debido a que la participación individual se puede mejorar en el escenario grupal. En ambientes de investigación bien seleccionados, los datos recolectados utilizando un grupo focal, pueden ser más informativos que los datos recopilados por otros métodos.

\section{LAS VICTIMAS DE LA VIOLENCIA Y EL TRABAJO DE INVESTIGACION}

En Colombia el conflicto ha propiciado múltiples variaciones e interrelaciones entre diversos actores armados, y no armados, signado por la violencia. Su recurrencia histórica es abordada por múltiples investigadores entre ellos: Carmen Lucia Díaz (2002, p. 107), quien precisa:

Reconocemos en toda esta problemática algo que retorna de manera insistente en la historia, que se repite en diversas épocas, de manera casi igual con actores distintos. Desde la conquista Colombia ha vivido situaciones de barbarie y de terror, efectuadas en un momento por el extranjero, ahora por el hermano, buscando siempre la expropiación y el aniquilamiento del otro, haciendo ilegitimo a quien tiene legitimo derecho y haciendo inoperante el orden regulador. Se ha impuesto el silenciamiento, el olvido, la orfandad, el destierro y la impotencia continúan, la falta de reconocimiento del otro se preserva. Tal vez la intervención de cada una de las instituciones siembra esperanzas para que la palabra del silenciado surja, para que el ocultamiento y el olvido secén, para que la legitimidad y reconocimiento retornen limitando el canibalismo del semejante por 
el más próximo; se advierte que aunque recuperar la palabra es fundamental, no todo puede quedar en el campo de lo simbólico, pues la afectación en lo real será necesario, jugando allí un papel fundamental la justicia en el reparto de los bienes y los privilegios.

La importancia de la Víctima se suscita como tema de investigación, por la complejidad que representa y por la relevancia que exhibe en la actualidad como centro para el desarrollo normativo. Podemos en ese sentido considerar la elocuencia de Víctor Guerrero (2007, p. 223) quien señala que:

(...) La víctima es inicialmente, y por lo menos hasta la década de los noventa, un ocupante sin lugar. Es aquello que está detrás de los procesos transicionales, pero que no tiene un lugar específico en los mecanismos judiciales para hacer valer su voz y para tener presencia como tal. El Tribunal de Nuremberg - referente obligado y punto de partida fundamental de toda esta tendencia política normativa y ética - juzgo a los más grandes criminales nazis. Sin embargo, ocho millones de víctimas judías circunscritas a ese ámbito racial, no fueron consideradas como tales.

Para Acosta (2004, p. 2), la víctima ha sido objeto de un tratamiento inadecuado, que justifica la realización de la presente investigación, objetivando el sentido de la misma para comprender las realidades y contrastar las investigaciones anteriores, es un punto de referencia que precisa ser contrastado y determinar de dicha aseveración los reales alcances, dado que el investigador asegura respecto de las víctimas, que han:

(...) sido sometidas a un injusto y desequilibrado estatuto del "olvido"; su presencia a lo largo del proceso se reduce al de mero objeto de valoración probatoria, como en el caso de las víctimas de violación o de lesiones, o en el mejor de los casos a un personaje secundario $e$ intrascendente que si mucho es acreedor de compasión de los operarios del sistema judicial y de la sociedad. La concepción de derecho... ha distanciado ostensiblemente el delincuente de la víctima y ha puesto a esta última como un simple sujeto pasivo, destinataria casual del crimen. De esta manera nuestro sistema penal ha "despojado" a la víctima de su carácter de sujeto de conflicto para ser sustituido de manera simbólica y abstracta por la comunidad, en el que el ofendido pierde su carácter de individuo para convertirse en multitud y perderse en una masa amorfa llamada comunidad o, como se le denomina en la praxis judicial, la sociedad.

Gutiérrez (2011, p. 3), en su referencia a las víctimas ha determinado que existen procesos inadecuados, sujetos de ser modificados para un real acceso a la justicia y para que:

\section{(...) tengan un espacio y un protagonismo} hasta ahora ausente, pero más que eso necesitan una sistema que conjugue el derecho a la justicia, a la verdad, a la reparación y a la participación. Estos cuatro elementos permiten que la víctima recupere la confianza, en sí misma, en el Estado-ley, y en los otros. La confianza, esencia central de las relaciones sociales, sin la cual la sociedad no podría existir y los grupos humanos se disgregarían... implica ante todo que frente a un proceso de victimización haya, en primer lugar la garantía de que la víctima podrá acceder a la justicia, y en segundo lugar que se hará realmente justicia. Este acceso a la justicia debe iniciar por generar mayor credibilidad en las instituciones encargadas de administrar justicia, brindándole a la víctima la seguridad de que sus denuncias serán atendidas con prontitud y respeto, evitando como ocurre frecuentemente, que se produzca una victimización secundaria.

Los textos consultados y transcritos permiten consolidar una situación problémica en torno 
a la víctima, que la presente investigación pretende abordar desde la psicología jurídica, para realizar en el Departamento del Magdalena una contrastación que sustente o no las teorías hasta ahora expuestas.

Además se debe estudiar y ordenar la dimensión del problema, para obtener reales diagnósticos y una solución acertada. Las dimensiones iniciales pueden ser consideradas según el planteamiento de Carolina Gutiérrez sobre el ordenamiento legal (2001, p. 2), como origen prístino:

(...) se ha enfocado en ver el delito como una infracción de la norma por parte de un individuo contra el Estado, en el que el infractor debe pagar una sanción para compensar el daño que ha causado a la sociedad, este es un proceso en el que la víctima, la comunidad y el mismo ofensor, desarrollan un papel pasivo y el daño de la víctima se compensa con un daño al ofensor. Por lo tanto se deben atender las necesidades de las víctimas $y$ del victimario, propendiendo por que este último se reincorpore a la sociedad asumiendo la responsabilidad de sus actos, crea además una respuesta al delito desde los mismos protagonistas, es decir que las víctimas se integran al proceso como seres propositivos y proactivos, no ya como los sujetos pasivos.

La participación activa de la víctima, empoderada y con un claro control de su vida, puede considerarse como un factor determinante para la reconstrucción de su entorno y de sus condiciones de vida. (Gutiérrez, 2011, p. 4):

(...) la participación como uno más de los derechos de las víctimas, comprendiendo que esto significa que la víctima pueda hacer parte de un proceso que conjugue a la verdad, la justicia y la reparación. Que se le permita a la víctima, tomar decisiones, formular propuestas y pasar del lugar sujeto pasivo, atado a un lugar de sufrimiento y olvido, a sujeto proactivo, propositivo, capaz de cambiar su propio destino.

El comprender la multidimensionalidad de la Víctima, su entorno y los procesos a los cuales está sujeta, son los argumentos principales que sustentan esta investigación y que evidencian la importancia de la psicología Jurídica.

\section{LA PSICOLOGIA JURIDICA Y LA VICTIMA. APORTES DE LA INVESTIGACION}

Los mejores avances en el pensamiento científico y los saltos paradigmáticos se dan en las fronteras entre ciencias, en los entrecruzamientos entre diversas ciencias que se abren a estudiar posibilidades no exploradas o no vistas por los limitantes señalados por los paradigmas establecidos (Khun, 1994). La ciencia sobre lo jurídico no puede estar excluida de estos procesos. Las sociedades son cambiantes y el derecho debe estar a tono con los cambios que operan en la sociedad estableciendo una dialéctica revitalizadora imparable. El derecho no se puede cerrar sobre sí mismo, como campo científico debe estar abierto, debe estar en un contexto, y debe nutrirse de la complejidad, de ahí que Letaif (2011, p. 1), conciba el derecho como una ciencia de lo contextual y de la apertura:

Un derecho- referencia, cerrado en sí mismo no garantiza la regulación de los modos de vida en la sociedad. La referencia se encuentra en el texto y el texto no es sin con-texto. La complejidad de la aplicación del Derecho en la regulación de la pulsión de muerte, está en conjugar principios referentes que en sí son contradictorios, tensos y disjuntos, en una sociedad compleja y plural y con modos de vida tan desiguales. La experiencia real exige un derecho abierto $y$ afectado por la contingencia, un derecho posible y plural que se orienta por un eje de principios. 
Por ello la realidad actual Colombiana, hace pertinente que se aborde lo jurídico desde una visión de la complejidad, del entrelazamiento entre campos del saber, de la multidisciplinariedad para generar transdisciplinariedad, o mejor aun para propiciar un conocimiento holístico y contextualizado.

La psicología es una ciencia de campos entrelazados. Que pugna por incluir otros campos de conocimientos a sus áreas de frontera científica. De ahí que nuevamente Letaif (2011, p. 1) nos llame la atención sobre como la psicología es un campo de saberes en construcción permanente, precisamente de saberes entrelazados:

La Psicología es una disciplina que se entrecruza con diversos campos del conocimiento, como la medicina, la antropología, el derecho, la economía. ... en cada una de esas intersecciones se genera un intrincado acervo de conocimientos; al entrecruzamiento entre el conocimiento psicológico y jurídico se denomina Psicología Jurídica.

Aunque de data reciente, la psicología jurídica es una ciencia que poco a poco ha materializado un campo de prácticas y un campo de investigación y desarrollo de conocimientos, donde se apoya al campo de lo jurídico. El entrecruzamiento entre lo jurídico y lo psicológico es entendido como psicología jurídica. Letaif (2011, p.3, 5) nuevamente aborda el entrecruzamiento de estos dos campos de conocimiento científico y se aventura a una definición de lo que es la psicología jurídica:

El contexto determina nuestras prácticas; $y$ ello también nos insta a una re definición de la Psicología Jurídica. (...) coincidimos en definir a la Psicología Jurídica como el conjunto genérico de la relación entre la psicología y el derecho; cubriendo todas las áreas de aplicación, se traten ellas de la psicología forense (que sería el área aplicada de la psicología jurídica, que relaciona a la utilización de los conocimientos psicológicos que son necesarios para ayudar a resolver un caso judicial en las áreas penal - familiar - laboral - civil ó dependientes del cuerpo de Psicólogos Forenses ó de las diversas oficinas periciales.) , la psicología criminológica (estudio de la conducta delictiva y antisocial, pero excluye todas las otras áreas de aplicación de la psicología al Derecho, como lo civil, lo laboral, etc.). Diremos que la Psicología Jurídica comprende el estudio, asesoramiento $e$ intervención eficaz, constructiva y prosocial sobre el comportamiento humano y las normas legales e instituciones que lo regulan.

En la era actual, una era de incertidumbre constante, lo interdisciplinario se convierte en la esencia de la psicología jurídica, así como su laboratorio experimental termina siendo el mundo de lo jurídico. La inserción paulatina de la psicología en el ámbito de lo jurídico, hace que se hibriden metodologías, retomen conceptos y se reorganicen procesos, que hacen de la psicología jurídica una ciencia que combina de forma sistemática subsistemas de pensamiento para poder aproximarse a certezas en medio de la cambiante sociedad, según Amar y Tirado (2011, p. 10):

... conjuga los planteamientos y hallazgos teóricos-experimentales propios de la psicología con las exigencias del quehacer teórico-práctico de las ciencias jurídicas; es la integración de dos subsistemas: el jurídico y el psicológico, por lo tanto... se puede afirmar que la psicología jurídica es un espacio interdisciplinario donde se utilizan y combinan conocimientos $y$ metodologías que le son propios.

Una de las áreas que se desprende de la psicología jurídica es la victimología. Espacio de investigación sobre las víctimas, sus necesidades, sus procesos de terapia y los diversos acompañamientos psicosociales que estas personas demandan. Según Tapias (2011, p. 3) el estudio de las víctimas desde la psicología jurídica implica:

Este nuevo apartado se dedica al estudio, prevención, tratamiento y asesoría 
(individual y grupal) a las víctimas del delito, para ayudarlas a reestablecerse y/o disminuir su potencialidad para convertirse en agresores. Esta área incluye la realización y análisis de encuestas de victimización fuera de las instituciones del estado para detectar cifras negras Soria, M.(1.993). También cobija la intervención en crisis para víctimas de delitos violentos, la asesoría a las víctimas para remitirlas según sus expectativas al sistema judicial, de protección o a asesoría psicológica, como es necesario en casos de violencia conyugal, asesoría psicojurídica para que las víctimas exijan el restablecimiento de sus derechos o la reparación por medios civiles como la indemnización, terapia de apoyo para víctimas indirectas como las familias de los secuestrados y asesoría al gobierno sobre políticas de manejo de grupos de víctimas de la violencia social.

La importancia de la Psicología Jurídica, que establece el horizonte hacia la cual se dirige esta investigación, la tornan en una actividad compleja y difícil, dada la sinergia de múltiples concepciones, acepciones y realidades:

El concepto de asistencia a las víctimas y los sinónimos relacionados (apoyo, defensa, asistencia, ayuda, servicios) generalmente tienen el significado de aplicación de una acción directa y personal dirigida a reducir el sufrimiento y a incrementar la recuperación de las víctimas de un delito. En un sentido más amplio, esto incluye actividades como restitución a las víctimas, derechos de las víctimas, compensación a las víctimas, información sobre el estado de los casos, grupos de apoyo, mediación y reconciliación entre víctima y agresor, asistencia telefónica, intervención en crisis, asesoramiento y terapia de las víctimas, servicios de emergencia médicos, servicios sociales, compañerismo, protección de victimización secundaria, por mencionar los más frecuentemente citados" (Dussich, 2001, p. 145).
Es en este aparte donde podemos vislumbrar el principal aporte que se hace desde la psicología jurídica a los procesos de acompañamiento psicosocial en las victimas. Es entender que los programas de asistencia a las victimas deben concebir la atención al trauma desde la asistencia única y personal, en correspondencia con sus condiciones particulares. No solo debe afrontarse de una forma especial con el individuo, sino que debe asumirse desde una óptica de restitución de los contextos sociales del sujeto que padece el trauma. El evento traumático es relevante para la toma de decisiones en torno a los programas de atención, Aristizábal (2011, p. 163):

El evento traumático no es causa material; la cuestión de saber eso que hace el trauma es específica a cada caso particular. Teniendo en cuenta esto, es necesario referirse a los testimonios de los sujetos para establecer eso que obro para ellos en calidad de traumatismo y ha generado un estado de vulneración psicológica a causa del encuentro con un hecho real que genero espanto, miedo o desagrado. Este encuentro con un horror psíquicamente inasimilable va a determinar que "el sujeto quede fijado a un fragmento del pasado [...] y en consecuencia este enajenado del presente y del futuro" (Freud, 1919).

Algunos investigadores y teóricos consideran muy importante propiciar los espacios adecuados para una atención ordenada a la víctima, su real restablecimiento y su proyecto vital. Es suministrar las herramientas de empoderamiento que le permitan el desarrollo de su proyecto de vida. Así entiende el empoderamiento Gutiérrez (2001, p. 2):

El empoderamiento... se entiende aquí como una serie de acciones encaminadas a que una persona adquiera, a través de un incremento en el acceso a los recursos (jueces, psicólogos, médicos, expedientes, restituciones), un control sobre su vida y sobre las decisiones que toma, en pro de su bienestar físico y mental, que a la 
vez redundará en beneficio de los que la rodean y de la comunidad en general. El empoderamiento es un proceso mediante el cual las personas incrementan su capacidad de configurar sus propias vidas.

Que coincide con la posición teórica de Villa:

(...) el primer paso para lograr la recuperación debe estar dirigido al empoderamiento de la persona, de su vida, que sienta y experimente que puede hacer algo y que el hecho violento no la paralizo; aun cuando haya implicado la pérdida de un ser querido, la mutilación de una parte de su cuerpo o el padecimiento de algún tipo de tortura o violencia psicológica. Es decir se debe trabajar hacia la dignificación de la persona, de su familia e incluso de la comunidad". (Villa Gómez; et al, 2007, p. 27).

La búsqueda de recursos internos es la meta final de un proceso psicosocial de empoderamiento de la ciudadanía tal y como lo plantea Gutiérrez (2011, p. 3):

(...) el empoderamiento debe llevar además a que las personas, encuentren sus propios recursos, se apropien de recursos externos y aprendan a utilizar ambos. Estos recursos pueden ser: Recursos Humanos: Como lo pueden ser el mediador, los jueces, la comunidad, el agresor, miembros de la familia, entre otros. Recursos físicos: establecimientos, transporte, etc. Recursos Psicológicos: confianza, auto-control, bienestar emocional, etc. Recursos intelectuales: información, ideas, conocimiento de situaciones, etc. Recursos financieros: salarios, donaciones, dinero, inversiones, etc".

Las personas empoderadas influyen positivamente en su entorno, se creen canales comunicativos asertivos entre los miembros de las comunidades afectadas, se desarrolle la confianza como la base de las relaciones interpersonales.
Otro de los aportes de la presente investigación, determina como contribución de la psicología jurídica a los procesos psicosociales de atención a las victimas los supuestos mismos del empoderamiento. Esto es referido al desarrollo de la proactividad. La proactividad encuentra su antecedente histórico más cercano en la Logoterapia instaurada por Víctor Frankl (2006).

De la Investigación puede desprenderse un campo de acción en el tratamiento y determinación del empoderamiento y de la proactividad de la víctima, entendido este último como el proceso de enseñarles a elegir como actuar frente a los estímulos del entorno, es decir, como responder mediante el uso adecuado de la libertad interior. Podemos elegir como sentirnos y si reflexionamos sobre el sentido de nuestras acciones podemos proyectarlas hacia el futuro (Fankl, 2006, p. 99):

“... al hombre se le puede arrebatar todo salvo una cosa: la última de las libertades humanas - la elección de la actitud personal ante un conjunto de circunstancias - para decidir su propio camino".

La proactividad es una herramienta valiosa para el despliegue de fortalezas interiores. Las personas proactivas deciden como sentirse frente a los estímulos externos, hacen un uso efectivo de su libertad interior y a cada momento se preguntan, que pueden aceptar de los estímulos externos brindando autoconciencia e inteligencia emocional. El empoderamiento permite un afianzamiento de la proactividad. Nos dice Covey (1997, p. 85):

Si bien la palabra proactividad es ahora muy común en los textos de dirección de empresas, se trata de un término que no se encuentra en la mayoría de los diccionarios. No significa solo tomar la iniciativa. Significa que, como seres humanos, somos responsables de nuestras propias vidas. Nuestra conducta es una función de nuestras decisiones, no de nuestras condiciones. Podemos subordinar los sentimientos a los valores. Tenemos la 
iniciativa y la responsabilidad de hacer que las cosas sucedan. Examinemos la palabra responsabilidad, en la cual encontramos alusiones a las palabras responder y habilidad: habilidad para elegir la respuesta. Las personas muy proactivas reconocen esa responsabilidad. No dicen que su conducta es la consecuencia de las condiciones, el condicionamiento o las circunstancias. Su conducta es un producto de su propia elección consciente; se basa en valores, y no es producto de las condiciones ni está fundada en el sentimiento.

Otro de los aportes de la investigación, subyacentes en la Psicología Jurídica tiene relación con el retorno de la víctima a su esencia proactiva, tornándose en una persona activa y consciente, con una gran posibilidad de refundar su memoria desde sus tragedias y desde sus esperanzas, tal y como ya lo había señalado Elizabeth Lira (2000b, p. 339) a propósito de la experiencia Chilena Post-Pinochet, cuando intuye la necesidad de convertir a la víctima en un sujeto que vuelva a ser sujeto y se ahuyente de la mirada compadecida que concibe a la víctima como un ser pasivo.

"El sujeto necesita volver a ser sujeto, ser considerado como persona, y no como víctima. No objeto de compasión. Necesita recuperar su dimensión de persona, activa, pensante, participativa. Persona al fin de cuentas. En síntesis, la palabra, la elaboración emocional de la experiencia traumática, su comprensión en el marco de la vida y las significaciones existenciales del sujeto posibilitan su rehabilitación como sujeto adulto, capaz de trabajar, de establecer vínculos sociales, desarrollando espacios de participación deseada y necesaria. Así, la experiencia traumática será parte de un contexto personal y social construido en un pasado significativo que apunta a un futuro capaz de ser desentrañado como un lugar de esperanza.
Finalmente uno de los aportes más importantes de la presente investigación es determinar el grado de construcción de resiliencia, como objeto de las actividades desarrolladas en la población objetivo.

La resiliencia es la forma como los seres humanos podemos reorganizarnos interiormente. El concepto de resiliencia explica la capacidad humana de superar traumas y dolores. El trabajo con las victimas arroja resultados empíricos que evidencian que los seres humanos no están aferrados a sus dolores interiores de por vida. A este desencadenarse, a este desamarrarse del dolor es a lo que se le denomina resiliencia. La resiliencia inicia cuando el ser humano es capaz de encontrarse a si mismo dándole un sentido a su existencia. Nos dice Boris Cirulnik (2006, p. 41) al respecto:

La resiliencia no se interesa más que en las formas de recomponer estos desgarros traumáticos. Pero, para pensar la resiliencia, es preciso convertir la propia historia en una visión en la que cada encuentro sea una elección existencial. Esta forma de dar un sentido no inexorable a la propia vida es expresión de una capacidad de libertad interior. Esta actitud abre la posibilidad de mil escenarios, con los titubeos, los golpes de suerte y las angustias que provoca toda elección. Las personas que razonan de este modo se sienten a gusto en las culturas de la incertidumbre, en las que pueden exponer su vida con facilidad. Esta pequeña libertad es una labor de artesanía en la que cada gesto y cada palabra pueden modificar la realidad que nos arrastra y construir la resiliencia como antidestino.

Importan a la investigación desde la perspectiva de la Psicología Jurídica, las singularidades del proceso de acompañamiento, el empoderamiento, la proactividad, la memoria significativa y trascendente, así como la resiliencia conceptos que emanan y nutren de los campos de la investigación científica de la psicología y el derecho, relacionada con la victimología. 


\section{CONCLUSIONES}

El ordenamiento jurídico propende por el restablecimiento de lo perdido, del estudio de los procesos de acompañamiento psicosocial, que puedan lograr en su empoderamiento a las víctimas.

Solo en la medida en que seamos capaces de asumir estos aportes en los procesos de acompañamiento, podremos retornar a unas condiciones reales y a una validez de la justicia social e incluyente, con efectividad simbólica y fáctica.

La psicología insiste en el camino de los símbolos para reconstruirnos interiormente, y el derecho entiende que la justicia solo podrá existir cuando esos símbolos de orientaciones interiores se tornan en pautas de referencia en las grandes representaciones colectivas. La norma solo es funcional cuando se tiene conciencia de la misma y eso ocurre cuando determina con precisión las personas a las que se dirige.

La norma ha establecido una serie de actividades para la atención a las víctimas, que deben ser evaluadas, valoradas y sujetas a permanente control, dado que se encuentran orientadas a la satisfacción de los cambios que de manera violenta han sido alterados en los destinatarios, quienes se encuentran en estados de permanente evolución.

La búsqueda de patrones exitosos, no puede convertirse en una constante de aplicación universal dada la diferencia de condiciones en la población destinataria y a sus condiciones de particularidad en la cual desarrollan sus proyectos de vida.

\section{BIBLIOGRAFÍA}

ACOSTA, Jairo (2004). La recomposición de las víctimas desde una perspectiva constitucional y humanitaria. Recuperado de http:// psicologiajuridica.org/archives/1285
ALVAREZ, Diego (2011). Desnudez en tres actos. En: SERNA DIMAS, Adrian. Territorios y desarraigos. Bogotá: Universidad Distrital Francisco José de Caldas.

AMAR, José; TIRADO, Diana (2011). Definiendo la psicología forense. En: ARISTIZABAL, Edith; AMAR, José. Psicología forense: estudio de la mente criminal. Barranquilla: ediciones UNINORTE.

ANRUP, Roland (2011). Antígona y Creonte: Rebeldía y Estado en Colombia. Bogotá: Ediciones B Colombia.

APONTE, Luz Stella (2007). Por la memoria, la dignidad y la esperanza. Aproximación a las expectativas de las víctimas del genocidio contra la Unión Patriótica. En: HOYOS VÁSQUEZ, Guillermo. Las víctimas frente a la búsqueda de la verdad y la reparación en Colombia. Bogotá: Editorial Pontificia Universidad Javeriana.

BUSHNELL, David. Colombia una Nación a pesar de sí misma. Bogotá: Planeta.

BONILLA, Elssy; RODRIGUEZ, Penélope (2005). Más allá del dilema de los métodos: la investigación en ciencias sociales. Bogotá: grupo editorial Norma.

CIRULNIK, Boris (2006). El amor que nos cura. Barcelona: Gedisa. Segunda edición.

COVEY, Stephen (1997). Los siete hábitos de la gente altamente efectiva. Barcelona: Paidos plural.

DIAZ, Carmen (2002). Destierro, desolación y reparación. En: BELLO, Martha Nubia. Efectos psicosociales y culturales del desplazamiento. Bogotá: Universidad Nacional de Colombia.

DUSSICH, J. (2001). Posibilidades de los programas de asistencia a las víctimas, en: Recuperación de las Víctimas, Opúsculos de derecho penal y criminología. Marcos Lerner Editora, Córdoba, Argentina.

FOREING POLICY (2011). Recuperado en: http:// www.fp-es.org/indice-de-estados-fallidos-2011 
FRANKL, Víctor (2006). El hombre en busca de sentido. Barcelona: Herder.

GARDINER, G. (2003). Discurso psicológico vs. Discurso jurídico. En: Construir puentes en psicología jurídica. Bs. As. JVE.

GUERRERO, Víctor (2007). La víctima: de ocupante sin lugar a lugar sin ocupantes. En: HOYOS VÁSQUEZ, Guillermo. Las víctimas frente a la búsqueda de la verdad y la reparación en Colombia. Bogotá: Editorial Pontificia Universidad Javeriana.

GUTIERREZ DE PIÑERES, Carolina (Marzo de 2011). El empoderamiento de las víctimas desde la justicia restaurativa. Recuperado de http:// psicologiajuridica.org/archives/1855.

HOYOS VÁSQUEZ, Guillermo (2007). Las víctimas frente a la búsqueda de la verdad y la reparación en Colombia. Bogotá: Editorial Pontificia Universidad Javeriana.

INFORME DE CARGA GLOBAL DE LA VIOLENCIA ARMADA (2011). Secretaria de la declaración de Ginebra sobre violencia armada y desarrollo.

KHUN, T.S. (1996). iQue son las revoluciones científicas. Barcelona: Altaya.

LATORRE, Edimer; CAMARGO, José Antonio; y BLANCO BOTERO, Carlos Armando (2010). Comunicación, memoria y resiliencia. Estudio sobre la memoria de las víctimas de la violencia en el Departamento del Magdalena. Bogotá: Fondo de publicaciones Universidad Sergio Arboleda.

LETAIF, G. (Enero, 2011). Psicología Jurídica en Con-texto. Recuperado de http:// psicologiajuridica.org/archives/885
LIRA KORNFELD, Elizabeth (2000 a). Psicología del miedo y conducta colectiva en Chile. En: MARTIN-BARO, Ignacio. Psicología social de la guerra: trauma y terapia. San Salvador: UCA editores.

LIRA KORNFELD, Elizabeth (2000 b). La tortura. Conceptualización psicológica y proceso terapeutico. En: MARTIN-BARO, Ignacio. Psicología social de la guerra: trauma y terapia. San Salvador: UCA editores.

MARTIN-BARO, Ignacio (2000). Psicología social de la guerra: trauma y terapia. San Salvador: UCA editores.

MORSE, Janice (2003). Asuntos críticos en los métodos de investigación cualitativa. Medellín: Editorial Universidad de Antioquia.

PEARSON, Annette (2006). La victimología y sus desarrollos en América Latina. La Gaceta, Numero 21. Bogotá: Fundación País Libre, Noviembre de 2006.

SANCHEZ, Natalie; et al (2011). Reparación psicosocial. Fundamentos para la reparación integral de personas víctimas del conflicto armado Colombiano. Bogotá: CINEP.

SELIGMAN, Martin (2002). La autentica felicidad. Barcelona: ediciones B.

TAPIAS, A. (Febrero, 2010). Aportes de la psicología jurídica a la criminología y al derecho. Recuperado de http://psicologiajuridica.org/ archives/239.

VILLA GOMEZ, Juan David; et al (2007). Nombrar lo innombrable. Reconciliación desde la perspectiva de las víctimas. Bogotá: CINEP. 